\title{
45th Winter School on Wave and Quantum Acoustics XLIVth Winter School on Environmental Acoustics and Vibroacoustics Szczyrk, Poland, February 29-March 4, 2016
}

On behalf of Upper Silesian Division of the Polish Acoustical Society (main organizer) and Committee of Acoustics of the Polish Academy of Sciences (coorganizer) we are pleased to announce that it is nearing the end of preparations for the conduct traditionally organized Conferences, which will take place as previously in Szczyrk. At the moment, more than 50 people have confirmed participation in Conferences. They will present more than 40 lectures and reports.

45th Winter School on Wave and Quantum Acoustics constitutes a platform for sharing the results and achievements obtained in different branches of physical acoustics (e.g. molecular acoustics, quantum acoustics, acousto-optics, magnetoacoustics, photoacoustics, acoustics of solid state etc.). Moreover, researches in some selected topics related to those mentioned above (e.g. optoelectronics, relaxation processes) will also be presented during the school. Currently, during the Conference Workshop on Acoustoelectronics and Workshop on Molecular Acoustics, Relaxation and Calorimetric Methods will be held - however, there is a possibility of organizing workshops on other subjects in the future. We would like to invite scientific centers and groups to cooperate in organizing workshops on the subjects of their interests.

XLIVth Winter School on Environmental Acoustics and Vibroacoustics is the forum for all environmental and vibroacoustics fields. Particularly it concerns traffic noise, vibroacoustics of machines, room acoustics, building acoustics, noise protection and similar problems.

In this issue one can find abstracts of some lectures, which will be presented during the Conferences. Further information about Conferences is available on our website

$$
\text { http://ogpta.pl/index.php/oddzial/ }
$$

Chairman of Organizing Committee

\author{
Abstracts \\ 12th Winter Workshop on Acoustoelectronics \\ Evaluation of the technical condition \\ of the active part of the high \\ power transformer based \\ on measurements and analyzing \\ of vibroacoustics signals \\ BORUCKI Sebastian, s.borucki@po.opole.pl \\ Cichó́ Andrzej \\ MaJCHRZAK Henryk \\ ZMARZŁY Dariusz \\ Institute of Electrical Power and Renewable Energy \\ Opole University of Technology \\ Prószkowska 76, 45-758 Opole, Poland
}

The subject of article refers to the results of research related to the development and industrial use of vibroacoustic methods for the assessment of the technical condition of the active part of transformers. The article presents results the analysis of the mechanical vibrations of the transformer high power, for which on the basis of chromatographic oil has been identified defect parts active. In order to confirm damage to the transformer active part vibroacoustic measurements were taken in three states work. Measurement using the classical vibroacoustic method include the registration of vibration in the idle state and the load operating of the transformer. During the measurements also used an author's diagnostic method, so called modified vibroacoustic method, which relies on the measurement and analysis of vibration of the transformer at the time of switching. The analysis shows that for the generation of elevated levels of mechanical vibration diagnosis transformer responds the magnetic circuit and the part of the windings vibration, caused by the current passing through it, is relatively insignificant. The presence of a narrow frequency band in the structure of vibroacoustic signal registered at the start-up of the transformer and the first few seconds of his unknown work, as well as a significant increase in the value of the components of band $5-1000 \mathrm{~Hz}$ for $t>4$ seconds, can attest to the gradual loss of the rigidity of the mechanical structure of its core. 


\section{Research and modeling of mechanical crosstalk in linear arrays of ultrasonic transducers}

CELmer Mateusz, mateusz.celmer@pwr.wroc.pl OPIELIŃSKI Krzysztof J.

Faculty of Electronics

Wrocław University of Technology

Wybrzeże Wyspiańskiego 27, 50-370 Wrocław, Poland

Linear arrays of ultrasonic transducers are commonly used as ultrasonic heads in medical diagnostics ultrasonography for imaging the interior of a human body in vivo. The crosstalk phenomenon occurs during the operation of transducers in which electrical voltages and mechanical vibrations are transmitted to adjacent components. As a result of such additional excitation of the transducers in the array, the directivity characteristics of the aperture used changes, and consequently there is interference with proper operation of a given array and the emergence of distortions in the reconstructed ultrasound image that reduce its quality. This paper studies the manner of propagation of mechanical crosstalk in a linear array of ultrasonic transducers on the basis of the recorded mechanical crosstalk, which appeared on elementary piezoelectric transducers when power is supplied to the selected transducer in the array. The paper includes a proposition of the model of a linear array of ultrasonic transducers and a simulation using finite element method (FEM) in order to confirm the results of the research.

\section{$\star \star \star$}

\section{Application of the Acoustic Emission Method in On-Load Tap Changer Diagnosis}

Cichoń Andrzej, a.cichon@po.opole.pl BORUCKI Sebastian

MajCHRzAK Henryk

Faculty of Electrical Engineering, Automatic Control and Computer Science

Opole University of Technology

Prószkowska 76, 45-758 Opole, Poland

This paper provides example of application of the acoustic emission (AE) method for diagnosis of technical condition of three-phase on-load tap-changer (OLTC) of GIII type. Measurements were performed for a population of 10 items of OLTCs, installed in power transformers with a capacity of 250 MVA. The study was conducted in two OLTC operating conditions: during the tapping process under load and free running conditions. The analysis of measurement results is presented in time domain and time - frequency. Description of AE signals generated by the OLTC in the time domain was performed using analysis of waveforms and determined characteristic times. Within time-frequency domain, measured signals were described by spectrograms for power density spectra by use of shorttime Fourier transform.

\section{Deposition of polymer sensor films on SAW surface by electrospraying technology}

GrabKa Michał $^{1}$, Neffe Sławomir ${ }^{1}$, JAsek Krzysztof ${ }^{1}$ PASTERnAK Mateusz ${ }^{2}$, mateusz.pasternak@wat.edu.pl

${ }^{1}$ Institute of Chemistry

Military University of Technology

S. Kalisliego 2, 00-908 Warszawa, Poland

${ }^{2}$ Institute of Radioelectronics

Military University of Technology

S. Kalisliego 2, 00-908 Warszawa, Poland

One of the most important problem in the surface acoustic wave (SAW) sensors technology is deposition of chemosensitive films having appropriate sorptive properties and morphology characterised by homogeneity, adhesion and durability high enough.

Very promising method of film deposition in this case seems to be electrospraying technology. It allows to obtain the chemosensitive layers of almost any chemicals and its mixtures in a controllable way. The control of the process parameters gives a possibility to deposit the specific films having properties unattainable by other methods.

In the paper the results of experiments with electrospraying in order to obtain of nafion film with platinum catalyst is described. In their framework the influence of the process parameters on film morphology and sensitivity have been studied.

The assessment of parameters of the selected ultrasonic medical devices and their conformity with the standards of the International Electrotechnical Commission (IEC)

Gudra Tadeusz, Tadeusz.Gudra@pwr.edu.pl Rosiak Agata

Faculty of Electronics, Wrocław University of Technology Wybrzeże Wyspiańskiego 27, 50-370 Wrocław, Poland

The paper presents the problem of compliance with international standards for ultrasonic medical diagnostic and therapeutic devices. The assessment of compliance of parameters of such devices has been carried out in relation to three standards: IEC 61157, IEC 61161, and IEC 61391. In particular, technical data contained in the documentation of four ultrasound devices used in medical diagnostics and five devices used in ultrasound therapy were analyzed. Data from the technical sheets of devices have been compared with the requirements of these standards, pointing out the lack of some important data in the cards of individual devices. It is especially disturbing that the manufacturers do not provide a lot of important data on the equipment used in ultrasound therapy and even the lack of specific technical sheets, which in practice makes it impossible to refer to the applicable IEC standards. The lack of a lot of information may result in exceeding the safe limit of ultrasound intensity that can cause damage to certain tissues in the body of a patient. 
Numerical analysis of non-steady stage in recovery step of the response of SAW to toxic gas

HeJczyK Tomasz ${ }^{1}$, thejczyk@ente.com.pl

WszoŁeK Bartłomiej ${ }^{1}$, JAKUBik Wiesław ${ }^{2}$

${ }^{1}$ ENTE Sp z o.o.

Gaudiego 7, 44-100 Gliwice, Poland

${ }^{2}$ Institute of Physics SUT

Konarskiego 22B, 44-100 Gliwice, Poland

The paper presents the results of numerical analyses of the SAW gas sensor in the steady and non-steady state. The effect of SAW velocity changes vs. the surface electrical conductivity of the sensing layer is predicted. The conductivity of the porous sensing layer above the piezoelectric waveguide depends on the profile of the diffused gas molecule concentration inside the layer. Knudsen's model of gas diffusion was used.

Numerical results for the gas $\mathrm{CH} 4$ for layers: WO3, $\mathrm{TiO} 2, \mathrm{NiO}, \mathrm{SnO} 2$ in the steady state and $\mathrm{CH} 4$ in the non-steady state in recovery step in the WO3 sensing layer have been shown. The main aim of the investigations was to study thin film interaction with target gases in the SAW sensor configuration based on simple reactiondiffusion equation.

The results of numerical analyzes allow to select the sensor design conditions, including the morphology of the sensor layer, its thickness, operating temperature and layer type. The numerical results basing on the code written in Python, are described and analyzed. The theoretical results were verified and confirmed experimentally.

\section{Analytical model of the acoustically loaded sandwich transducer}

\section{Kogut Paweł, Milewski Andrzej}

KARDYŚ Witold, KLuK Piotr

Tele and Radio Research Institute

Ratuszowa 11, 03-450, Warszawa, Poland

Ultrasonic transducers used in a high power applications such as ultrasonic welding or cleaning systems are mostly constructed as a piezoelectric stack systems known as sandwich transducers. Explicit formulation of the sandwich transducer parameters is well known and documented but only in reference to unloaded conditions and in parallel resonance working regime only. To overcome those difficulties many authors have been using lumped models such as Mason model or T-network model of the acoustic transmission lines as well as the finite element modeling. This approach takes time and obviously is less transparent than explicit formulation of the basic transducer parameters. This article provide an extent to the existing explicit formulations of the sandwich transducer parameters such as resonant frequencies, electromechanical coupling factor, mechanical amplitude gain factor and power gain factor. Authors derived explicit relations for those parameters taking into consideration acoustic loading conditions and both series and parallel resonant frequencies working regime. Designated formulas can be use to optimize transducer geometry dimensions such as piezoceramic stack length and po- sition in order to maximize the transducer acoustic power capabilities under certain acoustic loading medium. Obtained results have shown that acoustic loading and resonant frequency working regime have strong influence on the transducer parameters.

$$
\star \star \star
$$

Study on descriptors of acoustic emission signals generated by partial discharges under laboratory conditions and in on-site electrical power transformer

Kunicki Michał, CichoŃ Andrzej, a.cichon@po.opole.pl BORUCKI Sebastian

Faculty of Electrical Engineering, Automatic Control and Computer Science, Opole University of Technology Prószkowska 76, 45-758 Opole, Poland

An acoustic emission method (AE) is widespread and often applied for partial discharge (PD) diagnostics, mainly due to its ease of application as well as noninvasiveness and relatively high sensitivity. This paper presents comparative analysis of AE signals measurement results archived under laboratory conditions as well as on-site actual AE signals generated by inside PDs in electrical power transformer during its normal service.

Three different PD model sources are applied for laboratory research: point to point, multipoint to plate and surface type. A typical measuring set up commonly used for on-site transformer PD diagnostics is provided for the laboratory tasks: piezoelectric joint transducer, preamplifier, amplifier and measuring PC interface. During the onsite research there are three measuring tracks applied simultaneously. Time domain, time-frequency domain and statistical tools are used for registered AE signals analysis. A number of descriptors are proposed as a result of the analysis.

In the paper, attempt of $\mathrm{AE}$ signals descriptors, archived under laboratory condition application possibilities for on-site PD diagnostics of power transformers during normal service is made.

$$
\star \star \star
$$

\section{Comparison of low frequency signals emitted} by wind turbines of two different generator types

Malec Tomasz, Boczar Tomasz, t.boczar@po.opole.pl Wotzka Daria, Frącz Paweł

Institute of Electric Power and Renewable Technologies Opole University of Technology

Prószkowska 76, 45-758 Opole, Poland

Paper present results of comparative analysis of infrasound noise generated by wind turbines of two types: asynchronous type REPOWER MM92 with power equal to $2 \mathrm{MW}$ and synchronous type Vensys 62 with power equal to 1.2 MW. Frequency spectra of sound pressure levels generated during operation by both turbines for exemplary chosen wind speed values are depicted. Within the shown spectra the resonant frequencies have been indicated, for which sound pressure variations over time are shown. Based on 
the achieved frequency spectra it was stated that in general the asynchronous type turbine produces lower pressure levels, which are less stable over time, and indicates higher pressure values around the resonant frequencies as compared to the synchronous type turbine. Also it was stated that the asynchronous type turbine is more influenced by the wind conditions and generates higher pressure values by higher wind speeds then the synchronous type turbine.

$$
\star \star \star
$$

\section{Acoustic Emission as nondestructive testing method}

OLSZEwSKA Aneta, aneta.olszewska@polsl.pl

WiTos Franciszek, franciszek.witos@polsl.pl

Department of Optoelectronics

Faculty of Electrical Engineering

Silesian University of Technology

Krzywoustego 2, 44-100 Gliwice, Poland

Non-destructive testing is a group of testing methods that provide information about the properties of the object under test without causing damage. Common NDT methods include ultrasonic, magnetic-particle, radiographic or eddy-current testing give information only about local properties of the tested object. The method of acoustic emission testing (AT) due to its specificity stands out among the other NDT methods. AT method treats inspected object in a global manner. This technique is used to examination of structures, material study and control over manufacturing processes.

In this paper an overview of the application of AT method within petrochemical industry (pressure vessels, oil and gas pipelines, tanks), power distribution systems (i.e. transformers), railway transport and concrete constructions is presented. One of the most common application of AT method is metal pressure vessels inspection. The technique is valuable for detection of defects and damage during active load of tested object such as growth of cracks and other discontinuities in the structure of the material, local plastic deformation, material degradation due to corrosion causing local weakening of the structure of the material. Research methodology (equipment, arrangement of sensors, load curve, location of AE sources, classification of activity of AE sources) on the example of pressure vessels testing according standard PN-EN 14584:2013-07 is presented.

\section{Electromagnetic Interference shielding vs surface modifications - the review}

SzAFrAŃSKI Mateusz, mateusz.szafranski@wat.edu.pl KAwALEC Adam, adam.kawalec@wat.edu.pl DUKATA Andrzej, andrzej.dukata@wat.edu.pl OKOŃ-FĄFARA Marta, marta.okon@wat.edu.pl

Institute of Radioelectronics

Military University of Technology

Sylwestra Kaliskiego 2, 00-908 Warszawa, Poland

Even before Maxwell's equations were formed, humanity was exposed to numerous electromagnetic fields.
However it was not until 20th century when manmade electromagnetic fields became more and more abundant. Many systems and platforms are sources of unintentional EM fields, which are potentially harmful to other electronic hardware. On top of that, at some point, these emissions may reach power levels that pose serious threat even to human health.

This issue required a new branch of electromagnetic devices to be designed to protect against adverse emissions. Nowadays this branch bears the name of EMI shielding (Electromagnetic Interference - EMI). It covers every possible aspect of modifying original materials in order to increase their shielding effectiveness against electromagnetic fields. Furthermore it applies to gaskets, physical layers, that cover desired circuits and even special paints that absorb EM energy.

This paper presents the review of theoretical and practical works describing EMI shielding among various frequency bands. Special attention has been paid to the methods regarding surface modifications.

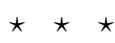

The computer measuring system

for registration

of acoustic emission signals

and location of acoustic

emission sources

within oil power transformers

SzERszeŃ Grzegorz, szerszen_g@yahoo.com

Witos Franciszek, franciszek.witos@polsl.pl

OpILSKI Zbigniew, zbigniew.opilski@polsl.pl

SETKIEwICZ Maciej, maciej.setkiewicz@polsl.pl

Department of Optoelectronics

Faculty of Electrical Engineering

Silesian University of Technology

Krzywoustego 2, 44-100 Gliwice, Poland

The computer measuring system, designed, built and called by Authors as $8 \mathrm{AE}-\mathrm{PD}$, dedicated for location and description of partial discharges within power oil transformers investigated by means of acoustic emission method is presented. Within the work detailed issues concerning the construction and operation of preamplifiers, instrumentation amplifiers, measurement card, magnetic holders of sensors, power supply and control system are described. The system is equipped with eight measurement channels and ensures provides monitoring of signals, data recording in the real time within the band of $20-1000 \mathrm{kHz}$ in laboratory and real conditions and as well the basic and advanced analysis of recorded data.

The measuring system has been calibrated and tested in laboratory conditions which were carried out with the use of modeled partial discharges sources. Obtained results confirm the high sensitivity of 8EA-PD measurement system and its usefulness in the study of partial discharges within oil power transformers. 
Analysis of the properties of acoustic emission signals generated in the pressure vessel during the hydraulic test

WiTos Franciszek, franciszek.witos@polsl.pl OPILSKI Zbigniew, zbigniew.opilski@polsl.pl OLsZEwska Aneta, aneta.olszewska@polsl.pl SETKIEwICZ Maciej, maciej.setkiewicz@polsl.pl

Department of Optoelectronics

Faculty of Electrical Engineering

Silesian University of Technology

Krzywoustego 2, 44-100 Gliwice, Poland

The results of analysis of the properties of acoustic emission signals recorded in the pressure vessel during a modified hydraulic test are shown. On the tested object, signals were recorded in two stages: preliminary and main. Within preliminary study, the set of curve attenuation and wave velocity of acoustic emission were carried out. From this, the EA sensor arrangement during the main research was determined. The main research has been carried out as part of the hydraulic test on the examined object. Course of hydraulic test has been modified by determining the course in two cycles in line with the requirements of the standard PN-EN 14584:2013-07. During main research, acoustic emission signals were recorded using the AMSY 6 Vallen system equipped with 30 tracks of measuring AE sensors arranged in the planned measuring points on the test object.

Properties of registered acoustic emission signals have been analyzed in terms of checking of Kaiser effect, the effect of felicity and integrity of the structure of the object. Location of acoustic emission sources active during the study and classification of these sources were carried out.

\section{2th Winter Workshop on Molecular Acoustics, Relaxation and Calorimetric Methods}

\section{Conductometric measurements \\ and their significance in the physicochemical studies of electrolyte solutions}

Boruń Agnieszka, chmielewska.a@gmail.com BALD Adam

Department of Physical Chemistry

University of Łódź

Pomorska 163, 90-236 Łódź, Poland

Conductometry is an electroanalytical method involving the measurement of electrolytic conductivity used both in research laboratories as well as in industry. It is one of the simplest, convenient, affordable and most accurate techniques for the investigation and analysis of substances and one of the most appropriate methods for studying of the nature of ion-ion, ion-solvent and solvent-solvent interactions existing in the electrolyte solutions.

It can also be used to determine the many physical quantities such as degree of dissociation, dissociation constants and solubility products. The data allow the calculation of thermodynamic functions of association, standard enthalpy of electrolyte solution, constants of complex formation, emf, free enthalpy of transfer, transfer numbers or the determination of the critical micellization concentration.

Evaluating the conductivity within the framework of present electrolyte theories requires high accuracy in measurements. In order to obtain the sufficiently accurate conductance data, which could be used in various conductance equations, it is necessary to have an equipment which allows reasonably accurate measurement at different temperatures.

The aim of this lecture is to discuss the theoretical and practical aspects of conductivity, the factors that influence the reliability of the measurement, and the techniques, as well as some applications of conductometry.

\section{Hydration of urea and its derivatives}

Burakowski Andrzej, Andrzej.burakowski@gmail.com GLIŃSKI Jacek

Faculty of Chemistry, University of Wrocław F. Joliot-Curie 14, 50-383 Wrocław, Poland

A series of dilute aqueous solutions of urea and its selected derivatives (1,3-dimethylurea, 1,1,3,3-tetramethylurea, 1,3-dimethylimidazolidin-2-one, and 1,3-dimethyltetrahydropyrimidin-2(1H)-one) was investigated by sound speed and density measurements.

The hydration numbers were determined using the compressibility data using Pasynski equation.

Molar volumes determined from density data of solutions were shown to reflect the hydration phenomena, too. Using this quantity, the contribution of carbonyl group, $>\mathrm{C}=\mathrm{O}$, to the total observed hydration number was determined.

\section{Halogen bonding in solution}

ChęCIŃSKA-MAJAK Dorota, dorotamajak@poczta.onet.pl BALD Adam

Department of Physical Chemistry, University of Lodz Pomorska 163, 90-236 Łódź, Poland

Halogen bonding (XB) is very commonly occurring force of long-range. It is a type of intermolecular noncovalent attractive interaction between a covalently bonded halogen atom and a negative site on an atom or group of atoms. Halogen bonding plays an important role in various scientific fields such as chemistry, biology, medicine or pharmacology. As the hydrogen bonding (HB) interaction is targeted with similar energy. Instead of a hydrogen atom, a halogen atom is collective to bond donor and acceptor. A characteristic property of this bonding is the directionality. The Y-X $\cdots \mathrm{B}$ bond angle in most cases close to $180^{\circ}$.

Many organic solvents are halogenated and a large number of reactions accur in halogenated solvents. Consequently halogen bonding should be expected in that cases. Up till now, studies of the halogen bonding in liquids were limited to theoretical and spectroscopic methods, and calorimetry. 
This lecture presents the study of tetrachloromethane $\left(\mathrm{CCl}_{4}\right)$ with diethylamine (DEA) mixtures. Densities $(\rho)$ and refractive index $(n)$ were measured as a function of mole fraction at $298.15 \mathrm{~K}$. The results were analyzed mainly in terms of the formation of intermolecular complexes by directional halogen bonds.

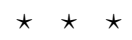

\section{Surface Properties of Chosen Task Specific Ionic Liquids - Measurements and Prediction}

GEPPERT-RYBCZyŃSKA Monika, monika.geppert-rybczynska@us.edu.pl

University of Silesia, Institute of Chemistry Szkolna 9, 40-006 Katowice, Poland

The surface tension, $\sigma$ of chosen sets of Ionic Liquids (ILs) based on imidazolium ring containing natural and/or pharmaceutical, or food compounds will be presented. The relation between surface tension and ILs structure will be discussed including influence of the length of alkyl side chain in cation in homologues series. A parachor concept, which assumes additivity in relation to constitution of ions, will be applied for $\sigma$ prediction. Additionally, the temperature dependence of surface tension for ILs will be analyzed and surface entropy will be calculated. All results will be discussed on the basis of comparison of correspondent quantities with those for the most popular ILs from among 1-alkyl-3-methylimidazolium tetrafluoroborates [CnC1im][BF4], bis(trifluoromethylsulfonyl)imides [CnC1im] [NTf2] and hexafluorophosphates [CnC1im][PF6] homologues series.

High pressure acoustic and thermodynamic properties of 2-methylfuran and 2,5-dimethylfuran - second generation biofuels

JĘżAK Sylwia

DzIDA Marzena

ZORĘBSKI Michał

Institute of Chemistry

University of Silesia

Szkolna 9, 40-006 Katowice, Poland

The two compounds of the furan family such as 2methylfuran (MF) and 2,5-dimethylfuran (DMF) are studied. These furan heterocycles can be obtained from cellulosic biomass and are indicated as a potential bioethanol or petroleum-based fuels substitute. The speeds of sound in MF and DMF were measured at temperatures from $293 \mathrm{~K}$ to $318 \mathrm{~K}$ and pressures from $0.1 \mathrm{MPa}$ to $101 \mathrm{MPa}$. The densities were measured for MF and DMF under atmospheric pressure in the temperature range from $273 \mathrm{~K}$ to $333 \mathrm{~K}$ and $273 \mathrm{~K}$ to $363 \mathrm{~K}$, respectively. The isobaric heat capacities were measured at atmospheric pressure and the temperature range from $293 \mathrm{~K}$ to $323 \mathrm{~K}$. The densities, isobaric heat capacities, isentropic compressibilities, isothermal compressibilities, and isobaric thermal expansions for temperatures from $293.15 \mathrm{~K}$ to $318.15 \mathrm{~K}$ and for pressures up to $100 \mathrm{MPa}$ were calculated using acoustic method. Differences between densities and isentropic compressibilities of MF and DMF can be compensated by changes of temperature and/or pressure.

$$
\star \star \star
$$

Ultrasonic studies of emulsion stability in the presence of magnetic nanoparticles

JózEFCZAK Arkadiusz, aras@amu.edu.pl ROZYNEK Zbigniew

Institute of Acoustics, Faculty of Physics Adam Mickiewicz University Umultowska 85, 61-614 Poznań, Poland

Superparamagnetic iron oxide nanoparticles (SPION) as well as composite materials based on them have unique features and many applications. An interesting application of these nanoparticles is to stabilize an emulsion. Solid magnetic nanoparticles are adsorbed strongly at the oilwater interface and are able to stabilize emulsions of oil and water. In this work emulsions stabilized by magnetite nanoparticles were obtained using high-energy ultrasound waves and a cavitation mechanism and, next, their stability in time was tested by means of acoustic waves with a low energy, without affecting the structure. An acoustic study showed high stability in time of magnetic emulsions stabilized by magnetite particles. The study also showed a strong influence of an external magnetic field, which can lead to changes of the emulsion properties. It is possible to control Pickering emulsion stability with the help of an external stimulus - a magnetic field.

\section{Application of the magnetic nanoparticles to enhance the effectiveness of the ultrasonic hyperthermia}

KACZMAREK Katarzyna ${ }^{1}$

JózEFCZAK Arkadiusz ${ }^{1}$, aras@amu.edu.pl Hornowski Tomasz ${ }^{1}$, KubovČíKOvÁ M. ${ }^{2}$

${ }^{1}$ Institute of Acoustics, Faculty of Physics Adam Mickiewicz University Umultowska 85, 61-614 Poznań, Poland

${ }^{2}$ Institute of Experimental Physics

Slovak Academy of Sciences

Watsonova 47, 04001 Košice, Slovakia

Ultrasonic hyperthermia is the method of cancer treatment in which tumors are exposed to elevated, cytotoxic temperature $\left(41-45^{\circ} \mathrm{C}\right)$ by means of ultrasound (US). In conventional ultrasonic hyperthermia the ultrasoundinduced heating in the tumor is achieved by the absorption of wave energy. However, to obtain appropriate temperature in reasonable time, the high US intensities, which can result in negative impact on healthy tissue, are required. The US effectiveness for medical purposes can be significantly improved by using the so-called sonosensitizers that can enhance the thermal effect of US on tissue by increasing US absorption. One possible candidate for such sonosensitiser are magnetic nanoparticles with mean sizes 
of $10-300 \mathrm{~nm}$ that can be efficiently heated due to additional attenuation and scattering of US.

$$
\star \star \star
$$

\section{Magnetically anisotropic composite systems}

KoPCANSKY Peter, kopcan@saske.sk

Tomasovicova Natalia, Gdovinova Veronika

Timko Milan

Institute of Experimental Physics

Slovak Academy of Sciences

Watsonova 47, 04001 Košice, Slovakia

This presentation deals with special class of magnetically anisotropic systems, i.e. composite systems consist of liquid crystals and magnetic nanoparticles, i.e. so-called ferronematics. These materials were introduced by Brochard de Gennes in 1970 to increase magnetic response of liquid crystals to external magnetic fields. Main aim is to prepare liquid crystalline sensors of magnetic field, i.e. magnetovision camera.

This contribution will present basic theory with description of these materials as well as many experimental data regarding structural changes so called Fredericksz transitions, response of these system to low magnetic field, nematic isotropic transition in presence of magnetic field as well as the idea how to prepare liquid ferromagnets. Nematic-isotropic phase transition of these systems in the presence of very small magnetic field opens the doors towards application possibilities such as low magnetic field sensors or basic logical elements for information storage technologies.

\section{$\star \star \star$}

Effect of temperature on acoustic and volumetric properties of aqueous mixtures of ionic liquids

MusiaŁ Małgorzata, gosiamusial19_91@interia.pl GePPERT-RyBCZyŃsKA Monika, ZoręBSKI Edward

University of Silesia, Institute of Chemistry

Szkolna 9, 40-006 Katowice, Poland

Based on the literature speed of sound and density data, the excess speed of sound, excess molar volume and excess molar isentropic compressibility of binary systems containing ionic liquid and water were calculated. All binary systems are completely miscible with water at the analyzed temperatures $(288.15 \mathrm{~K}, 298.15 \mathrm{~K}$ and $308.15 \mathrm{~K})$. The binary systems with water were chosen because of their importance and possible application (e.g. as absorption refrigeration systems). Some patterns of behavior for speed of sound and calculated quantities with respect to temperature and cation/anion structure are found. For example, for binary systems of similar ILs with water an inversion of the speed of sound isotherms was found (near IL molar fraction 0.05). Another interesting feature of some systems is that speed of sound changes significantly with concentration only at lower IL molar fraction, but for higher IL molar fraction resembles speed of sound of pure ionic liquid.

\section{Investigation of liquid crystals \\ - biphenyl derivatives}

PONIKWICKI Nikodem

LINDE Bogumił B.J., fizbl@ug.edu.pl

Institute of Experimental Physics

University of Gdańsk

Wita Stwosza 57, 80-309 Gdańsk, Poland

Liquid crystals selected for experiments were biphenyl derivatives: 4-alkyl-4'-cyanobiphenyls (nCB), which form a homologous series described by the formula $\mathrm{C}_{n} \mathrm{H}_{2 n+1^{-}}$ $\mathrm{C}_{6} \mathrm{H}_{4}-\mathrm{C}_{6} \mathrm{H}_{4}-\mathrm{CN}$. They are built of two subunits (phenyl rings linked by a $\mathrm{C}-\mathrm{C}$ bond) that can rotate about each other.

One of the phenyl rings in 4-alkyl-4'-cyanobiphenyl contains a cyano group $(-\mathrm{CN})$ substituted in $p$-position. Other molecules of the series differ in the length of their alkyl chains substituted in $p$-position at the second phenyl ring.

The nCB series have typical liquid crystalline phase sequences depending on chain length: for $n=5,6,7$ crystalnematic-isotropic, $n=8,9,11$ crystal-smectic A-nematicisotropic, $n=10,12$ crystal-smectic A-isotropic.

There were observed monotropic transition into nematic phase for $n=3$ and 4 , while cooling as well as photoacoustic spectra.

These molecules can adopt various conformations due to varying dihedral angle between the phenyl rings and different positions of the alkyl chain (all-trans, zig-zag conformation).

Experimental results reported in the literature are concerned with the measurements of the angle between phenyl rings, the effect of the alkyl chain length on the infrared spectrum, and the dipole moment of alkylcyanobiphenyls. The effect of different polar and nonpolar solvents on vibrational and electronic spectra was also investigated.

$$
\star \star \star
$$

\section{FT-EoS: data processing for determination of its parameters}

Postnikov Eugene B., postnicov@gmail.com NedYalkov Yuriy V.

Department of Theoretical Physics

Kursk State University

Radishcheva 33, 305000 Kursk, Russia

The Fluctuation Theory-based Tait-like Equation of State (FT-EoS) is recently proposed [Int. J. Thermophys. 35 (2014) 2115; Ind. Eng. Chem. Res. 54 (2015) 9645] as an effective isothermal equation of state, which does not require fitting experimental data for each isotherm in order to determine multiple sets of EoS's constants. Instead, on can utilize well-established correlations for the coexistence curve or operate with the fits of data obtained at the normal pressure.

We review available procedures, their numerical realizations and required data: samples of (i) the density and the isothermal compressibility at normal pressure; (ii) the speed of sound and the heat capacity ratio at normal pressure or along the coexistence curve, (iii) the normal boiling point temperature and an approximation of the saturated 
density. They are illustrated by the examples of normal and halogenated alkanes, aromatic hydrocarbons for the pressure ranges up to hundreds MPa. The developed Excel's user build-in functions suitable for practical applications will be provided.

The work is supported by RFBR, project number 1608-01203.

\section{$\star \star \star$}

Mechanical properties and instability of Pickering droplets probed by electric field induced stress and ultrasonic spectroscopy

ROZYNEK Zbigniew ${ }^{1,2}$, zbigniew.rozynek@gmail.com MikKelsen Alexander ${ }^{2}$, Dommersnes Paul ${ }^{2}$ JózeFCzAK Arkadiusz ${ }^{1}$, FOSSUM Jon Otto ${ }^{2}$

${ }^{1}$ Institute of Acoustics, Faculty of Physics

Adam Mickiewicz University

Umultowska 85, 61-614 Poznań, Poland

${ }^{2}$ Department of Physics, NTNU

7050 Trondheim, Norway

Recently, studies of particle laden droplets subjected to external electric fields has increased significantly with promising development in fields like electro-coalescence, destabilization of Pickering emulsions, or arrested coalescence. Here we study both the behavior of microparticle stabilized droplets in electric fields and formation of Pickering droplets via electro-coalescence. Colloidal capsules (composed of jammed microparticles) are made on a surface of oil droplets. The investigation concerns the viscoelastic deformation, shell unjamming, and solid-to-liquid transition of monolayered shell, and dynamics of shell formation. A new unreported method of fabricating Pickering shells is demonstrated. We wish to study the dynamics of formation of Pickering emulsion by indirect methods, e.g. ultrasonic spectroscopy, which may reveal the phase transition of the surface of a droplet (from liquid to solid like, when the droplet is fully covered by particles).

The effect of temperature on acoustic and volumetric properties of diluted solutions of water in ionic liquids

SKowroneK Justyna ${ }^{1}$, DzIDA Marzena ${ }^{1}$

ZoręBSKI Edward ${ }^{1}$, GePperT-RYBCZyŃska Monika ${ }^{1}$

JACQUEMIN Johan ${ }^{2}$, Goodrich Peter ${ }^{2}$

${ }^{1}$ Institute of Chemistry, University of Silesia

Szkolna 9, 40-006 Katowice, Poland

${ }^{2}$ The QUILL Research Centre

School of Chemistry and Chemical Engineering

Queen's University of Belfast

Stranmillis Road, Belfast BT9 5AG, United Kingdom

Acoustic and volumetric properties of diluted solutions of water in 1-butyl-3-methylimidazolium bis[(trifluoromethyl)sulfonyl]imide, $\left[\mathrm{C}_{4} \mathrm{mim}\right]\left[\mathrm{NTf}_{2}\right]$, 1-butyl-1methylpyrrolidinium bis[(trifluoromethyl)sulfonyl]imide, $\left[\mathrm{C}_{4} \mathrm{mpyr}\right]\left[\mathrm{NTf}_{2}\right]$, 1-propyl-3-methylimidazolium bis[(trifluoromethyl)sulfonyl]imide, $\left[\mathrm{C}_{3} \mathrm{mim}\right]\left[\mathrm{NTf}_{2}\right]$ and 1-ethyl3-methylimidazolium thiocyanate, $\left[\mathrm{C}_{2} \mathrm{mim}\right][\mathrm{SCN}]$ were investigated. The speeds of sound were measured within the temperatures from 293.15 to $318.15 \mathrm{~K}$. The densities were measured in the temperature range from 293.15 to $328.15 \mathrm{~K}$ for $\left[\mathrm{C}_{4} \mathrm{mim}\right]\left[\mathrm{NTf}_{2}\right]$ and 293.15 to $353.15 \mathrm{~K}$ for the remaining ILs. On the basis of experimental results the molar isentropic compressibility, the molar thermal expansion, partial molar volume, and partial molar adiabatic compressibility were calculated. The molar isentropic compressibilities and the molar thermal expansions of water + $\left[\mathrm{C}_{4} \mathrm{mim}\right]\left[\mathrm{NTf}_{2}\right]$ and water $+\left[\mathrm{C}_{4} \mathrm{mpyr}\right]\left[\mathrm{NTf}_{2}\right]$ solutions are approximately the same in the whole temperature range. The molar isentropic compressibilities and the molar thermal expansions of water $+\left[\mathrm{C}_{2} \mathrm{mim}\right][\mathrm{SCN}]$ solutions are the lowest among the investigated water + IL systems.

\section{Hyperthermic effect in mechanically treated magnetosomes}

Tıмko Milan, timko@saske.sk

Molcan Matus, Rajnak Michal, Kopcansky Peter

Institute of Experimental Physics

Slovak Academy of Sciences

Watsonova 47, 04001 Košice, Slovakia

The existence of biocompatible phospholipid membrane around magnetosome and the high value of specific absorption rate (SAR) predetermined this bacterial nanoparticles for the biomedical applications. The magnetosomes were prepared by biomineralization process of magnetotactic bacteria Magnetospirillum sp.AMB-1.The magnetosome chains (IM) extracted from the body of bacteria were divergent in length, i.e. chains of magnetosomes (IM) have been modified due to mechanical effects during sonication (SM). In this contribution we present the possibility of magnetosome chains characterization via small-angles techniques and the influence length of chains on the hyperthermia effect.

Experimental scattering curves of magnetosome IM and SM samples, indicate the presence of polydisperse particles in the investigated samples. The values of initial scattering intensities confirm the differences in average sizes of two kinds of samples prepared by various methods. This fact causes that SM sample magnetically behaves in a different meaner, showing that energy loss and specific absorption rate are noticeable reduced, and thereby indicates variation in the relaxation process and heat distribution and so can be used for hyperthermia application.

\section{Kinetics of acoustic relaxation processes in aromatic hydrocarbons and their halogensubstituted}

VERVEYKO Vyacheslav N., verveykovn@mail.ru VERVEYKO Marina V.

Research Center for Condensed Matter Physics Kursk State University Radishcheva 33, 305000 Kursk, Russia

Recently, there are different and sometimes contradictory points of view on the kinetics of acoustic relaxation 
processes in aromatic hydrocarbons and, especially, their halogensubstituted.

We discuss the results of our measurements related to the speed of ultrasound and its absorption rate at the frequencies $5-110 \mathrm{MHz}$, and the shear viscosity, the ratio of bulk and shear viscosities and relaxation times within the temperature range $293-373 \mathrm{~K}$ and the pressures $0.1-$ $50 \mathrm{MPa}$ and their dependences on PVT parameters and the frequency.

This analysis allows the conclusion that the vibrational relaxation is absent in these liquids within the investigated range of state parameters, which contradicts the previously accepted point of view. The kinetic mechanism for such a behaviour can be assumed, most probably as the processes of restructuring related to the reactions of rupture and formation of weak chemical bonds such as $\mathrm{C} \ldots \mathrm{H}-\mathrm{C}, \mathrm{C} \ldots \mathrm{H}-$ $\mathrm{Cl}, \mathrm{C} \ldots \mathrm{H}-\mathrm{Br}$ with formation energy about $4-6 \mathrm{~kJ} / \mathrm{mol}$.

This work was supported RFBR, project number 1608-01203.

\section{$\star \star \star$}

\section{XLIVth Winter School on Environmental Acoustics and Vibroacoustics}

\section{On some problems of LDV vibration measurements} for large windows

KWAPISZ Leszek ${ }^{1}$, kwapi@imp.gda.pl

RADZIEŃSKI Maciej ${ }^{1}$

JAKUBOWSKI Piotr ${ }^{2}$

${ }^{1}$ Institute of Fluid-Flow Machinery

Polish Academy of Sciences

Fiszera 14, 80-231 Gdańsk, Poland

2 Ship Design and Research Centre

Szczecińska 65, 80-392 Gdańsk, Poland

LDV (Laser Doppler vibrometer) can be a powerful non-contact tool in vibro-acoustic analyze of windows that allows the registration form of vibrations, of a window through which the sound waves pass by. This is of some relevance in research of acoustic windows insulating.
The vibrometers LDV measurements of large windows difficulties may arise in the investigation. The method which gives the most information is to sweep a large number of points on the window with the same excitation periodic chirp signal. This requires a few hours. In our research for a large window for the 7663 grid points $(97 \cdot 79)$ at the end of measurement used loudspeakers have shown signs of overheating and lost its full power of broadcasting. The results showed a step change in amplitude. To obtain reliable results for the whole window applied corrections, involving rescaling the results obtained with lower power of overheated speakers. Details and results will be given in the paper.

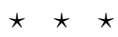

Noise and infrasonic noise at workplaces in wind turbines vicinity

Pleban Dariusz,daple@ciop.pl Radosz Jan, Smagowska Bożena

Central Institute for Labour Protection

- National Research Institute (CIOP-PIB)

Czerniakowska 16, 00-701 Warszawa, Poland

The entry into force in Poland of the act relating renewable energy sources, implementing the European directive $2009 / 28 / \mathrm{EC}$ on the promotion of the use of energy from renewable sources, should speed up investments in wind energy in Poland. The development of wind energy in Poland is accompanied by not only an increase in the number of people employed in the workplaces for the benefit of the energy, but also an increase in the number of people whose jobs are located in the vicinity of wind farms. An example of measurements and assessments of noise and infrasonic noise at selected work stations in wind farm vicinity is presented in the paper. The results of the measurements carried out at workplaces located inside and outside buildings at distances from 500 up to $2300 \mathrm{~m}$ of wind turbines VESTAS V80-2.0 MW did not exceed the values of Maximum Admissible Intensities for noise and the values of infrasonic noise nuisance criteria. 